\title{
Educação em saúde na perspectiva da Educação Física: impactos do comportamento sedentário na vida de escolares
}

\author{
Health education from the perspective of Physical Education: impacts of sedentary behavior in \\ schoolchildren's lives
}

La educación para la salud desde la perspectiva de la Educación Física: impactos del comportamiento sedentario en la vida de los escolares

\author{
José Giovanny Santos Andrade \\ ORCID: https://orcid.org/0000-0002-5611-0044 \\ Centro Universitário AGES, Brasil \\ E-mail: pef.j.giovanny@gmail.com \\ Humberto Costa Silva \\ ORCID: https://orcid.org/0000-0002-3472-4897 \\ Centro Universitário AGES, Brasil \\ E-mail: humberto.costa.berto@hotmail.com \\ Josefa Imara Santana Ribeiro \\ ORCID: https://orcid.org/0000-0002-0142-1937 \\ Centro Universitário AGES, Brasil \\ E-mail: ymararibeiro4@gmail.com \\ Luan de Jesus Faustino \\ ORCID: https://orcid.org/0000-0003-1148-4218 \\ Centro Universitário AGES, Brasil \\ E-mail: luanziinho.black@gmail.com \\ Cleiton Antonio de Oliveira \\ ORCID: https://orcid.org/0000-0002-0502-9262 \\ Centro Universitário AGES, Brasil \\ E-mail: cleiton.oliveira@ages.edu.br
}

\begin{abstract}
Resumo
Este estudo trata-se de uma revisão bibliográfica cujo objetivo é discutir os impactos do comportamento sedentário na vida de escolares, apresentando possíveis soluções para diminuir a problemática e consequentemente contribuir com a saúde pública positiva. Para isso, utilizamos trabalhos publicados no período de 2017 a 2021 disponíveis nas plataformas Scielo e Google Acadêmico. Dessa forma, faz-se um paralelo entre sedentarismo e obesidade, contextualizados pela Covid-19, apresentando as implicações no surgimento de doenças crônicas. Mediante assim, o estudo visa expor possíveis atitudes capazes de diminuir os níveis de inatividade física, frisando a importância da Educação Física no contexto escolar e extraescolar, pois evidencia-se que esta é uma das formas de conscientizar os alunos a adotar hábitos saudáveis que refletirão nas condutas positivas do futuro, sendo um importante meio de incentivar a prática de atividade física, tendo em vista que, a obesidade e a tendência ao comportamento sedentário não é apenas dominante no público adulto e idoso, mas também é uma problemática que abrange o público de jovens escolares.
\end{abstract}

Palavras-chave: Comportamento sedentário; Obesidade; Educação física; Atividade física.

\begin{abstract}
This study is a literature review which aims to discuss the impacts of sedentary behavior in schoolchildren lives, presenting possible solutions to reduce the problem and, consequently, contribute to positive public health. For this, we used works published from 2017 to 2021 available on the Scielo and Google Academic platforms. Therefore, it is established a parallel between sedentary lifestyle and obesity, contextualized by Covid-19, presenting the implications for the emergence of chronic diseases. Accordingly, the study aims to display possible attitudes capable of reducing levels of physical inactivity, emphasizing the importance of Physical Education in the school and out-of-school context, as it is evident that this is one of the ways to educate students to adopt healthy habits that will reflect in the positive behaviors of the future, being an important way to encourage the practice of physical activity, considering that obesity and the tendency to sedentary behavior is not only dominant in adults and elderly, but it is also a problem that affects the public of young students.
\end{abstract}

Keywords: Sedentary behavior; Obesity; Physical education; Physical activity. 


\begin{abstract}
Resumen
Este estudio es una revisión bibliográfica cuyo objetivo es discutir los impactos del comportamiento sedentario en la vida de los escolares, presentando posibles soluciones para reducir el problema y, en consecuencia, contribuir a una salud pública positiva. Para ello, se utilizaron trabajos publicados en el periodo comprendido entre 2017 y 2021 disponibles en las plataformas Scielo y Google Acadêmico. Así, se establece un paralelismo entre el sedentarismo y la obesidad, contextualizado por Covid-19, presentando las implicaciones en la aparición de enfermedades crónicas. Así, el estudio pretende exponer posibles actitudes capaces de reducir los niveles de inactividad física, destacando la importancia de la Educación Física en el contexto escolar y extraescolar, ya que es evidente que ésta es una de las formas de concienciar a los alumnos para que adopten hábitos saludables que se reflejen en las conductas positivas del futuro, siendo una forma importante de incentivar la práctica de la actividad física, teniendo en cuenta que la obesidad y la tendencia al comportamiento sedentario no sólo es dominante en el público adulto y anciano, sino que también es un problema que abarca al público de jóvenes estudiantes.
\end{abstract}

Palabras clave: Comportamiento sedentario; Obesidad; Educación física; Actividad física.

\title{
1. Introdução
}

O comportamento sedentário tem revelado um contexto preocupante no Brasil, tendo em vista o quantitativo da população classificada como sedentária ou com baixos níveis de atividade física. Nesse tocante, percebe-se que os brasileiros têm permanecido muito mais tempo em atividades de repouso, ao contrário do tempo ativo, conforme recomenda a (Organização Mundial da Saúde [OMS], 2020). Isso é evidenciado em dados expressivos, mostrando que $71 \%$ a $78 \%$ do público infantojuvenil está em inatividade (Santana, 2021). Essa problemática tem se estendido também ao público de adultos e idosos, pois segundo os dados divulgados em 2019 pela Pesquisa Nacional de Saúde (PNS), proporcionalmente, mais de 40\% dessa primeira população, e mais de $59 \%$ da segunda, não estão suficientemente ativas com base nos propósitos da OMS (Instituto Brasileiro de Geografia e Estatística [IBGE], 2020).

Nessa direção, observa-se que os baixos níveis de atividade física contribuem na ocorrência de várias doenças crônicas, como obesidade, diabetes, doenças cardiovasculares e câncer (Nahas, 2017). Logo, percebe-se que isso vem preocupando as autoridades de saúde devido ao nível de exposição à comorbidades em um ritmo frequente. Dentre essas, a obesidade tem sido alarmante devido ao seu grau de interferência nas demais patologias (Ferreira \& Andrade, 2021). Para tanto, Andrade e Batista (2021) salientam que o IBGE em 2020 mensurou o número de obesos cuja porcentagem ultrapassa os $60 \%$ do quantitativo da sociedade de brasileiros, como também aponta dados que abrangem todas as faixas etárias.

No entanto, embora essa inquietação esteja em evidência e seja de suma importância, é preciso levar em consideração que o atual contexto pandêmico causado pelo vírus da Covid-19 também favoreceu o aumento dos índices de pessoas com pouca exercitação em 14\% para o público masculino e mais de 10\% em relação ao feminino (Malta et al., 2020). As orientações emitidas pela Organização Mundial de Saúde para o distanciamento social como permanência em casa, a fim de conter a propagação do vírus, tem influenciado as pessoas a terem condutas pouco saudáveis, o que inclui a falta ou a redução da prática de atividade física e exercício físico, levando assim ao sedentarismo (Botero et al., 2021). Diante disso, ratifica-se que os dados adquiridos por instrumentos de pesquisa apresentaram uma queda de 15,3\% a 20,4\% no nível de atividade física no Brasil (Matsudo et al., 2020).

À vista da relevância desta revisão, é perceptível que a prevalência do comportamento sedentário está relacionada a várias doenças crônicas não transmissíveis, de modo que a pouca realização de atividade física e a obesidade associam-se diretamente com Doenças Cardiovasculares (DCVs), as quais causam distúrbio no coração e vasos sanguíneos (Correia et al. 2021). Sendo assim, a atividade física se mostra como contribuinte positivo na transformação do estilo e qualidade de vida da população, atrelando-se diretamente aos parâmetros biológicos (Dantas \& Silva. 2021). Por isso, acredita-se que o estímulo à vida ativa, desde as primeiras idades, pode contribuir para adoção de hábitos saudáveis ao longo da vida e diminuir os riscos associados à mortalidade e morbidade. 
Research, Society and Development, v. 11, n. 1, e25011124731, 2022

(CC BY 4.0) | ISSN 2525-3409 | DOI: http://dx.doi.org/10.33448/rsd-v11i1.24731

Os comportamentos sedentários estão cada dia mais nítidos na sociedade e não se trata de um contexto atual. O fato é que este aumento é frequente e a população brasileira tem mais pessoas em estilo de vida sedentário e com doenças crônicas diversas, as quais afetam diretamente na saúde e qualidade de vida, sem distinguir crianças, adolescentes e/ou idosos (Ferreira \& Andrade, 2021; Santana et al., 2021; Vasconcellos et al., 2021). No entanto, a preocupação que tem movimentado diversos estudos na atualidade é motivada pelo quantitativo de comorbidades, como obesidade, hipertensão e câncer, advindas dos hábitos de vida negativos (Ferreira et al., 2021). Nesse sentido, surge o questionamento que norteia a presente pesquisa: quais os impactos do comportamento sedentário na vida dos indivíduos e as medidas necessárias para o enfrentamento desta problemática?

Sendo assim, este artigo tem como objetivo principal discutir os impactos do comportamento sedentário na vida de escolares, apresentando possíveis soluções para diminuir a problemática e consequentemente contribuir com a saúde pública positiva. Para isso, de forma específica, pretende-se identificar os impactos causados por hábitos sedentários; discutir a relação entre estilo de vida sedentário e obesidade, bem como o papel de ambas no surgimento de doenças crônicas; correlacionar o contexto atual de comportamento sedentário e obesidade com a pandemia de Covid-19 para verificar possíveis problemas que podem vir a surgir em função dos hábitos inadequados em um período prolongado; por fim, apresentar possíveis atitudes capazes de diminuir os níveis de inatividade física.

Portanto, acredita-se que, por meio desta revisão bibliográfica, é possível cooperar com a ciência e consequentemente, por meio destes conhecimentos, provocar novas pesquisas, bem como alertar a população a respeito dos riscos atrelados aos hábitos não saudáveis ocasionados pelo baixo índice de pessoas ativas. Logo, com suas particularidades, a Educação Física, por meio de seus profissionais capacitados, pode contribuir positivamente neste contexto, visto que as práticas corporais trabalhadas sejam em ambientes formais ou informais, são capazes de incentivar as diferentes faixas etárias à prática de atividade física e/ou exercício físico, bem como a manutenção da vida saudável (Andrade \& Batista, 2021).

\section{Metodologia}

Esse artigo é de natureza aplicada, sendo ela caracterizada "por buscar a solução de problemas concretos, práticos e operacionais" (Zanella, 2009 como citado em Jacobsen, 2017, p.4), visto que serão discutidos os contextos que circundam o tema do sedentarismo. Nesse contexto, o foco está atrelado à discussão, frente aos impactos do comportamento sedentário na vida da sociedade e a partir desse ponto, lançar estratégias de prevenção e promoção da vida saudável, tendo o contexto escolar, por meio dos profissionais de Educação Física, como um dos caminhos essenciais para o enfrentamento a esta problemática, caracterizando-se assim como estudo dedutivo, pois serão analisados dados gerais em detrimento de uma situação específica (Lima, 2019).

Diante disso, o objetivo se dá por meio da pesquisa exploratória em busca de esclarecimentos e desenvolvimento de pensamentos (Lima, 2019), pois o tema em questão versa sobre os impactos do comportamento sedentário, que, em consonância com a pandemia da Covid-19, tem gerado novas discussões e perspectivas. Assim, para alcançar os objetivos desse tema, será fundamental realizar um estudo de cunho bibliográfico, ou seja, será levado em consideração e tomado como parâmetro, os conhecimentos já publicados (Sousa et al., 2021), os quais foram priorizados nesta produção, artigos entre 2017 e 2021, entretanto, o parâmetro temporal não se emprega a documentos de lei, Livros e E-books. Enquanto à abordagem, optou-se por uma análise qualitativa, considerando as diversas informações encontradas em prol da resolução da problemática, apontada na pergunta de pesquisa (Patias \& Hohendorff, 2019).

Nessa perspectiva, realizou-se a avaliação de 50 artigos, dos quais foram incluídos 26, além da Base Nacional Comum Curricular (BNCC) mencionada como Brasil (2017) e da Constituição Federal do Brasil (1998). Logo, para o embasamento 
Research, Society and Development, v. 11, n. 1, e25011124731, 2022

(CC BY 4.0) | ISSN 2525-3409 | DOI: http://dx.doi.org/10.33448/rsd-v11i1.24731

dos resultados e discussões amparou-se em critérios de elegibilidade, sendo feito uma análise do resumo dos artigos, resultando assim na inclusão deles. No entanto, após seleção, também foi avaliado todo o corpo de texto com intuito de identificar a relevância da bibliografia para o presente trabalho, visto que ele precisava versar com os temas (sedentarismo e suas problemáticas, influência da Covid-19 no comportamento sedentário, meios e/ou projetos de enfrentamento à inatividade física e sedentarismo no contexto escolar). E como mecanismos de busca, utilizou-se as plataformas Google Acadêmico e Scielo, a partir dos Descritores de Ciências da Saúde (DeCS/MeSH): Comportamento sedentário; Obesidade; Comportamento de risco à saúde; Doenças não transmissíveis; Exercício físico. Para além disso, também foram realizadas pesquisas por meio de palavras-chave: Educação Física e sedentarismo; Atividade física e sedentarismo; Sedentarismo e pandemia de Covid-19; Educação Física escolar e sedentarismo.

\section{Resultados e Discussão}

Os resultados deste trabalho possuem papel fundamental para comprovar a tese de que a sociedade brasileira está sujeita aos impactos causados por hábitos sedentários, mas também busca sustentar a ideia de que, quando é realizado um trabalho de prevenção e promoção à saúde desde as primeiras idades, principalmente, dentro do ambiente escolar, é possível facilitar o aumento dos índices de vida saudável. Também se discute a relação entre sedentarismo e obesidade, verificando o papel de ambas no surgimento de doenças crônicas; o contexto atual de sedentarismo e obesidade em decorrência da pandemia de Covid-19. Por fim, estudos que apresentassem possíveis atitudes capazes de diminuir os níveis de inatividade física.

Sobre os impactos causados por hábitos sedentários, iniciaremos a abordagem trazendo o conceito de comportamento sedentário. Ele pode ser definido como a falta ou diminuição de atividade física, estando em situações de baixo gasto energético, como é o caso da pessoa que está reclinada, deitada ou sentada. Este é um tema que, ao longo dos anos, vem sendo amplamente discutido pelos meios midiáticos e principalmente pelos profissionais de saúde. Isso porque o estilo de vida sedentário está relacionado a fatores de risco para o desenvolvimento ou até mesmo o agravamento de patologias (Benedetti et al., 2021). Assim sendo, é apontado como um problema contemporâneo da sociedade, visto que é classificado como a quarta causa de morte mundial com alta influência no que diz respeito ao surgimento de várias patologias, estando relacionado diretamente entre cerca de $3,8 \%$ a $5,5 \%$ de índice de mortalidade. Logo a seguir, serão discutidos os três primeiros, à hipertensão, representando 12,8\%, tabagismo 8,7\% e hiperglicemia 5,8\% (Raimundo et al., 2019).

Desse modo, é de suma importância salientar que este comportamento junto a outras problemáticas como a obesidade, configuram-se como um dos fatores preditores de doenças crônicas e não transmissíveis, dentre elas, o câncer, a diabetes e doenças cardíacas, afetando todos os estratos sociais. Neste ínterim, deve ser ressaltado também que a inatividade física é classificada como responsável por $7 \%$ de diabetes tipo 2, 6\% da ocorrência de doenças cardiovasculares, $10 \%$ de câncer da mama e 10\% de câncer do cólon (Raimundo et al., 2019).

Os estilos de vida sedentários, segundo Souza e Silva (2018), é também um fator agravante para evolução da obesidade. Os autores ainda frisam que esta comorbidade é tratada como uma questão de saúde pública, pois leva em consideração o seu custo, prevalência e efeitos à saúde, já que esta aumenta a probabilidade da ocorrência de várias outras problemáticas, dentre elas as doenças renais, cardiovasculares, diabetes, além de desencadear problemas respiratórios, como interferir também na diminuição da força muscular. Corroborando com esse viés, Who (2017) alerta que essas Doenças Crônicas Não Transmissíveis (DCNTs), na contemporaneidade, são uma das principais causas de óbito em todo o mundo.

Não obstante, os baixos níveis de atividade física apresentam-se como um fator significativo no aumento do colesterol LDL, no sangue, que nada mais é do que uma lipoproteína de baixa densidade que tem como principal função transportar o colesterol do fígado e de outros locais do corpo humano para as artérias. Mediante esta função, pode ocasionar um entupimento 
das artérias e provocar doenças como aterosclerose e infarto. Já o colesterol HDL é uma lipoproteína de alta densidade, que tem como finalidade transportar o colesterol das artérias para o fígado, para sua eliminação do organismo, evitando dessa forma seu acúmulo (Faludi et al., 2017).

Visto que, em tempos de Covid-19, o qual vem afetando todo o mundo, e, principalmente o Brasil, desde dezembro de 2019 foi declarado emergência de saúde pública. A partir de então, a sociedade vem lutando contra esse vírus que consegue se disseminar através do contato direto entre as pessoas, mais especificamente através da respiração e da fala. Como forma de enfrentamento, foram adotadas estratégias de isolamento e afastamento social em todo o Brasil, bem como em vários outros países do mundo. Essas estratégias visam o controle da disseminação viral, e principalmente o controle da mortalidade (Brandão, 2020).

O isolamento social, apesar de ser o caminho mais fácil no combate à Covid-19, pode se tornar um pesadelo para a saúde da população, em relação a alguns fatores, principalmente àqueles ligados a adoção de estilos de vida negativos que favorecem o aumento no número de obesos e do comportamento sedentário. A obesidade nada mais é do que uma doença multifatorial, cuja caracterização é o acúmulo de gordura corporal, que, em longo prazo, gera ou contribui para inúmeras outras doenças como: diabetes, hipertensão, câncer, e até mesmo se torna um agravante para a Covid-19, entre outros (Brandão, 2020).

Logo, as ações que buscavam isolar a sociedade para protege-los, culminou no fechamento de entidades comerciais, escolas e espaços destinados às práticas de exercícios diminuindo a propagação do vírus. Por outro lado, tais medidas vão de encontro ao combate à obesidade, gerando consequências como a privação da liberdade, resultando em mais tempo dentro de casa, diminuição das atividades físicas, bem como de atividades de lazer. Fatores esses que trazem complicações futuras à sociedade. A prática de exercício físico é algo indispensável no combate à Covid-19, pois atua no sistema imunológico de forma que minimiza os danos causados, sejam eles, físico, social ou psicológico, e combate à obesidade principalmente no público juvenil (Daronco et al., 2021).

Assim, as medidas tomadas no que diziam respeito ao confinamento, a fim de alcançar alguns objetivos como o controle viral e a mortalidade, acabam gerando outros agravantes como o comportamento sedentário em excesso, distúrbios alimentares (incluindo uma má alimentação), alterações psicológicas e fisiológicas, aumento no número de pessoas com ansiedade e até mesmo depressão, aumento no número do alcoolismo, tabagismo e inúmeras outras doenças, as quais trazem situações negativas para todo o País, em longo prazo (Daronco et al., 2021).

A pandemia da covid-19 trouxe consigo um impacto capaz de intensificar os problemas de saúde já existentes. Logo, os profissionais e sistemas de saúde encontram-se fragilizados, bem como a população, temendo o contato social e realizando o distanciamento, agravando significativamente outros problemas de saúde e tornando-o mais precário no sistema em geral. Esses abalos nos sistemas de saúde, bem como todos os problemas sociais e econômicos, podem levar ao abalo dos programas de imunização, aqueles que, no passado, já vinham lutando, pressionando governos e agências de saúde em geral, na última década (Couto et al., 2021).

Além disso, a pandemia não afetou gradativamente apenas o sistema de saúde, como também gerou um grande impacto no contexto educacional mundial. Assim, no que diz respeito aos impactos negativos do comprometimento do processo de ensino-aprendizagem e pelo número crescente de abandono escolar, demandam ações e estratégias emergenciais para que os alunos voltem e tenham interesse pelos estudos. Logo, cabe um esforço a mais, bem como um planejamento de resolução e normalização dos ciclos escolares a curto e médio prazo (Senhoras, 2020).

Para tanto, é necessária uma reeducação para que a saúde pública não venha a ficar mais frágil do que já está. É necessário que haja incentivo e modificação no estilo de vida, de forma imediata e em longo prazo, tanto para minimizar os comprometimentos da Covid-19, quanto em relação às sequelas que ela deixou na qualidade do estilo de vida da sociedade, 
pois a obesidade é considerada um fator de risco modificável, caso os indivíduos sejam orientados o quanto antes, e mudem seus hábitos. Visando a crescente prática de atividades físicas e uma boa alimentação saudável, essas devem ser a prioridade das políticas públicas de saúde e de educação (Bolsoni-Lopes et al., 2020).

Diante do exposto até o momento, torna-se perceptível que os hábitos negativos de saúde como os que levam os indivíduos a comportamentos sedentários têm influenciado diretamente na vida de crianças, jovens e consequentemente também interfere na vida adulta, pois este ciclo contínuo está relacionado aos hábitos adquiridos ao longo da vida os quais se enraízam como parte geradora de uma nova cultura. Assim, o incentivo contínuo às práticas corporais tem papel fundamental na formação de cada sujeito, ao passo em que os tornam seres autônomos e constituídos de culturas positivas, capazes de tornálos habituados na busca constante da vida ativa (Andrade \& Batista, 2021).

Neste ínterim, ações como criação de programas comunitários de atividade física, parcerias entre setores públicos e privados, bem como a manutenção das aulas de Educação Física no espaço escolar, tem se mostrado essenciais para diminuir os índices de sedentarismo, visto que, durante uma parcela do dia e durante a vida, o público infantojuvenil se encontra no ambiente educacional, possibilitando que o trabalho de conscientização a respeito da adesão de hábitos saudáveis seja realizado desde as primeiras idades e durante toda sua vida de estudante. Esta é apontada como uma das melhores alternativas, pois inúmeras vezes são nessas aulas que ocorrem os primeiros contatos com atividades estruturadas (Bottcher, 2019; Foppa \& Fernandes, 2020; Silva et al., 2021).

Destarte, é preciso que, para além do ensino-aprendizagem da Educação Física, as quais, atualmente no Brasil, possibilitam no máximo 2 horas por semana, essas crianças e jovens também se mantenham praticando atividade física e/ou exercício físico ao longo da semana, bem como nos horários extraescolares (Gomes et al., 2019; Salgado, 2017). Isso poderá ser feito por meio de políticas públicas de esporte e lazer com a perspectiva educativa, motivacional e ampliadora, gerando possibilidades para que engajem toda a população mantendo-os ativos, baseando-se primordialmente nos conhecimentos culturais de cada localidade, pois desta forma é observado mais efetividade (Bramante et al., 2020).

A partir disso, espera-se que esses alunos possam continuar priorizando a vida saudável, de modo que a realização de atividade física seja um dos meios promotores e preventivos de saúde. Esta que, apesar das diversas definições, pode ser compreendida através de pilares (social, emocional, espiritual, intelectual e físico), mas que se entrelaçam e fortalecem a necessidade do incentivo às práticas corporais (brincadeiras e jogos, esporte, lutas, ginásticas, danças e práticas corporais de aventura), bem como a atenção a cada indivíduo, proporcionando vivências que atuam na formação integral, necessitando que os profissionais também estejam preparados para identificar quaisquer contextos de saúde negativa, pois na atualidade, muito dos infantojuvenis têm apresentado alterações psicológicas, as quais têm influenciado nos outros pilares (Brasil, 2017; Campanhã et al., 2021; Garcia et al., 2015).

Vale ressaltar que no tocante aos transtornos psicológicos, há inúmeros fatores que se associam à pandemia da Covid19 e, principalmente, a opressão imposta pela sociedade dominante acerca da preparação para o mundo laboral, ou seja, crianças e jovens têm sido pressionados a saírem das escolas já preparados para trabalhar e isso tem sido notado na constituição dos currículos de alguns estados ao enfatizarem a inserção da formação técnica (Brioshi \& Bueno, 2021). Com isso, ressaltamos a importância dos cuidados para com este público e o amadurecimento de ideias que possibilitem a cada criança e jovem a convivência com condutas saudáveis, desde as primeiras idades, por meio das aulas de Educação Física.

O comportamento sedentário, no que concerne aos baixos níveis de atividade física, influencia no surgimento das diversas doenças já mencionadas neste artigo, sendo verdadeira a afirmação de que tais problemas não têm afetado apenas adultos, mas também adolescentes, ou seja, jovens em idade escolar. Desta forma, é factível a tamanha importância de reforçar e ampliar o quantitativo de programas e projetos que ponham o público infantojuvenil no centro dos estímulos voltados aos hábitos sadios, em virtude de sua vulnerabilidade social frente ao surgimento de variados apetrechos tecnológicos capazes de 
desestimular a vida ativa, uma vez que a adoção da saúde positiva desde as primeiras idades pode ser perpetuada (Carvalho et al., 2021; Voser et al., 2017).

Sendo assim, é fato que, dentro do meio educativo, está a maior possibilidade de incentivar, promover, possibilitar e proporcionar a constituição de um sujeito que cultue e preze por hábitos saudáveis, pois a escola é um ambiente em que as interações ocorrem de forma transversal e, neste contexto, permite o surgimento de iniciativas responsáveis por estimular as práticas de saúde inclusive de forma intersetorial entre as secretarias municipais (Silva et al., 2021; Voser et al., 2017). Logo, sabe-se que a Educação Física é responsável por facilitar o acesso dos alunos às práticas corporais, bem como discussão sobre temáticas transversais atreladas à saúde e lazer (Brasil, 2017). Embora, ainda seja necessário reivindicar que os membros do executivo e legislativo cumpram a Lei 9696/98, na qual estabelece os profissionais de Educação Física como responsáveis pela atuação nesses ambientes que visam à promoção da prática de atividade física e exercício físico. (Brasil 1998).

Para, além disso, percebe-se a importância da existência de programas paralelos, a fim de garantir a continuidade da promoção de atividade física, após momentos de aulas, bem como após a vida escolar, podendo ser citadas políticas públicas como "Programa Cidade Ativa, Cidade Saudável" (Silva et al., 2021, p.3), "Programa Academia da Cidade", "Programa Academia da Saúde" (Silva et al., 2021, p.2) e demais programas que podem ser encontrados no site do Ministério da Cidadania na pasta referente ao Esporte e Lazer, aos quais destaca-se o Programa Esporte e Lazer da Cidade. Portanto, são meios capazes de estimular o enfrentamento à inatividade física e, por consequência, o sedentarismo, pois possibilita acesso às práticas de atividades e exercícios físicos.

\section{Considerações Finais}

Por todo o exposto, é perceptível que o sedentarismo é um dos principais responsáveis pelo elevado índice de pessoas com obesidade e sobrepeso, mostrando-se junto a isso, a necessidade de trabalhar frente a essa temática no ambiente escolar, com o intuito de conscientizar e incentivar a adoção de hábitos saudáveis e, sobretudo, possibilitar aos alunos, atividades físicas como uma forma de diminuir os índices de sedentarismo, pois, constatou-se que a obesidade e tendência de sedentarismo não é exclusividade de adultos e idosos, mas sim, uma problemática que abrange o público de jovens escolares. Da mesma forma, ressalta-se a importância da implementação de políticas públicas que se adequem a este objetivo, bem como se reivindica que a Educação Física tenha, no mínimo, duas aulas semanais e, no máximo, uma aula diária com profissionais de formação nessa área.

O comportamento sedentário, em resumo, é a falta de exercícios e uma vida pouco ativa, associada à ingestão de comidas não saudáveis, gerando assim um acúmulo de gorduras em determinadas regiões do corpo, levando ao ganho mais rápido de peso, bem como o aumento de problemas de saúde. Dentre eles podem ser citados o aumento do colesterol, diabetes tipo 2, além do risco de derrame, problemas no coração, obesidade, entre outros. Para combater esses possíveis problemas, deve-se investir em hábitos de alimentação saudável e rotinas de atividade física, de preferência acompanhada por um profissional de Educação Física.

Ademais, para dirimir o sedentarismo, acredita-se que é importante a adoção de estratégias capazes de despertar cada vez mais o interesse da sociedade pelo estilo de vida saudável, seja através da conscientização dos indivíduos, facilidade ao acesso às práticas de atividades físicas desde cedo, seja dentro ou fora do cenário escolar, como também entende-se que o ingresso gratuito a estas práticas corporais nas escolas e em locais públicos, é um fator primordial, aliando-se à melhoria da infraestrutura, de modo que possibilite a locomoção e proporcione prazer às pessoas, ao poder desfrutar uma vida saudável ao ar livre. Ou seja, necessita-se de mais políticas públicas que reforcem o combate ao sedentarismo e demais fatores, mantendo a população em uma rotina de vida ativa e saudável. 
Aliado a isso, cabe ressaltar que a Covid-19 se tornou um grande influenciador de forma direta e indireta, do comportamento sedentário, como o aumento da obesidade, gerando nesse contexto, um cenário de vulnerabilidade social. Assim, a pandemia em nosso país, está entre uma das principais responsáveis pelo aumento no número de pessoas sedentárias, de obesos e de morbimortalidade. Logo, é importante ressaltar a expectativa de que, em longo prazo, e com a sociedade vivenciando um cenário de imunização através da vacinação da Covid-19, seja possível possibilitar mais acesso a exercícios físicos e a reabertura dos espaços voltados à efetivação de forma segura. Somando-se a isso, vale salientar que o profissional de educação física é o único responsável e preparado para promover e prescrever exercícios para todos os espaços, seja na sua modalidade on-line ou presencial.

Para tanto, por meio desta revisão torna-se perceptível a necessidade de chamar a atenção de todos para as problemáticas advindas dos comportamentos sedentários, ao passo em que reafirma a necessidade de investimento em espaços e políticas que possibilitem à sociedade, adotar o estilo de vida ativa e saudável de forma gratuita, destacando a escola como um dos principais pilares capazes de contribuir positivamente com a saúde individual e coletiva. Com isso, espera-se que sejam tomadas atitudes com intuito de legitimar, de forma organizada e responsável, as aulas de Educação Física escolar, ou seja, com tempo adequado e baseando-se nos tempos estimados pela Organização Mundial da Saúde e com supervisão de profissionais registrados no Conselho de Educação Física.

\section{Agradecimentos}

Agradecemos a todos que direta e indiretamente contribuíram para a realização e sucesso do artigo.

\section{Referências}

Andrade, J. G. S. \& Batista, M. S. B. (2021). As práticas corporais como contribuição preventiva à saúde nos cuidados com a obesidade infanto-juvenil. In F. L. O. de Carvalho, G. S. Dosea \& D. M. Costa. (Org.). Saúde Baseada em Evidências. Centro Universitário AGES, 1, $34-38$.

Benedetti, T. R. B., Borges, L. J., Streit, I. A., Garcia, L. M. T., Manta, S. W., Mendonça, G., Binotto, M. A., Christofoletti, M., Silva F.L. Jr., Hallal, P. C. \& Papini, C.B. (2021). Validade e clareza dos conceitos e terminologias do Guia de Atividade Física para a População Brasileira. Rev. Bras. Ativ. Fís. Saúde. 26, 1-11. $10.12820 /$ rbafs. $26 \mathrm{e} 0212$

Bolsoni-Lopes, A., Furieri, L. B., \& Alonso-Vale, M. I. C. (2020). Obesidade e a covid-19: uma reflexão sobre a relação entre as pandemias. Revista Gaúcha de Enfermagem, 42, 1-6. https://doi.org/10.1590/1983-1447.2021.20200216

Bottcher, L. B. (2019). Atividade Física como ação para promoção da saúde: Um ensaio crítico. Rev. Gestão \& Saúde (Brasília) Edição Especial, fev., 98-111. https://doi.org/10.26512/gs.v0i0.23324

Bramante, A. C., Andrade, D. R., Pitanga, F. J. G., Costa, L. P., Oliveira, L. C., Porto, L. G. G., Ferreira, M. B. R., Nahas, M. V., Santos. M. \& Matsudo, V. K. R. (2020). Manifesto Internacional para a Promoção da Atividade Física no Pós-COVID-19: Urgência de uma Chamada para a Ação. Rev. Bras. Ativ. Fís. Saúde. 24, 1-5. 10.12820/rbafs.25e0175.

Brandão, S. C. S., Godoi, E. T. A., Cordeiro, L. H. O., Bezerra, C. S., Ramos, J. O. X., Arruda, G. F. A. \& Lins, E. (2020). Obesidade e risco de COVID-19 grave. Simone Brandão. https://repositorio.ufpe.br/bitstream/123456789/37572/1/Obesidade\%20e\%20risco\%20de\%20Covid\%2019\%20grave.pdf

Base Nacional Comum Curricular: educação é a base. (2017). Ministério da Educação.

Brioschi, F. R. \& Bueno, C. R. Jr. (2021). Fatores ambientais na obesidade infantil. Rev. Conhecimento em Destaque, 7 (18), $95-115$.

Campanhã, L. M. F., Silva, M. L. M. P., Junior, A. T., Claro, R. F. T. \& Zanini, G. S. (2021). Educação física escolar e os efeitos nas variações de humor dos estudantes - uma revisão narrativa. Revista CPAQV - Centro de Pesquisas Avançadas em Qualidade de Vida, 13 (2), 1-8. 10.36692/v13n2-13R

Carvalho, A.S., Abdalla, P. P., Silva, N. G. F., Júnior, J. R. G., Mantovani, A. M. \& Ramos, N. C. (2021). Exercício físico e seus benefícios para a saúde das crianças: uma revisão narrativa. Revista CPAQV - Centro de Pesquisas Avançadas em Qualidade de Vida, 13 (1), 2-16. 10.36692/v13n1-7r

Couto, M. T., Barbieri, C. L. A. \& Matos, C. C. S. A. (2021). Considerações sobre o impacto da covid-19 na relação indivíduo-sociedade: da hesitação vacinal ao clamor por uma vacina. Saúde soc. 30 (1), 1-11.

Correia, A. P., Borges, F. J. S., Lima, K. O., Santana, K. M., Rodrigues. L. C. C. \& Borges, G. F. (2021). Ação extensionista de promoção da saúde e prevenção de doenças cardiovasculares para alunos de uma escola da Bahia. R. Eletr. de Extensão, 18(39), $218-227$.

Dantas, E. H. M. \& Silva, J. R. V. (2021). Atividade física, saúde e qualidade de vida. Atena. 
Daronco, L. S. E., Pozzobon, D., Ramos, D. L., Oliveira, J. M. S., \& Berria, J. (2021). Educação física e saúde em tempos de COVID-19. Observatório socioeconômico da COVID-19, Fapergs, 1-12. https://www.ufsm.br/app/uploads/sites/820/2021/04/Textos-para-Discussao-27-Educacao-Fisica-e-Saude-emTempos-de-COVID.pdf

Faludi, A. A., Izar, M. C. O., Saraiva, J. F. K., Chacra, A. P. M., Bianco, H. T., Afiune Neto A., Bertolami, A., Pereira, A. C., Lottenberg, A. M., Sposito, A. C., Chagas, A. C. P., Casella-Filho, A., Simão, A. F., Alencar Filho, A. C., Caramelli, B., Magalhães, C. C., Magnoni, D., Negrão, C.E., Ferreira, C. E. S., \& Salgado Filho, W. (2017). Atualização da diretriz brasileira de dislipidemias e prevenção da Aterosclerose. Arquivos Brasileiros de Cardiologia, 109(2) 1-76.

Ferreira, C. S. \& Andrade, F. B. (2021). Desigualdades socioeconômicas associadas ao excesso de peso e sedentarismo em adolescentes brasileiros. Ciência \& Saúde Coletiva, 26(3), 1095-1104

Ferreira, T. C. S. \& França, T. L. (2021). Contribuições da educação física na prevenção e tratamento da obesidade infantil. Scire Salutis. v.11(1), 7-23. DOI: http://doi.org/10.6008/CBPC2236-9600.2021.001.0002

Ferreira, T. S., Sales, A. F. S. \& Baptista, A. S. (2021). Exercícios físicos na prevenção de doenças crônicas não transmissíveis. Revista Saúde em Foco, v.13, $180-196$.

Foppa, D. L. \& Fernandes, M. M. (2020). Impacto da atividade física nos transtornos comportamentais em adolescentes [artigo graduação, Universidade Cesumar]. http://rdu.unicesumar.edu.br/bitstream/123456789/7802/1/FOPPA\%2c\%20Dara\%20Luiza\%3b\%20FERNANDES\%2c\%20Marcia\%20Maria.pdf

Garcia, P. T., Oliveira, A. E. F., Freitas, C. V. S., Baesse, D. C. L., Júnior, E. F. C. \& Pinho, J. R. O. (Org.). (2015). Saúde e sociedade. EDUFMA.

Gomes, J. A. Dimer, E., Pires, M., Alves, S. (2019). Participação dos Alunos com Sobrepeso nas Aulas de Educação Física Escolar. Trajetória Multicursos, 11(1), 32-48.

Jacobsen, A. L., Conto, S. F., Silvério, R. C., Guimarães, V. R. \& Silva, W. C. (novembro de 20217). Perfil metodológico de pesquisas elaboradas no âmbito das instituições de ensino superior brasileiras: uma análise de publicações feitas pela revista ciências da administração. Artigo apresentado no XVII Colóquio Internacional de Gestão Universitária, Mar del Plata, Argentina.

Lei n. 6.880, 9.696, de 1 de setembro de 1998 (1998). Dispõe sobre a regulamentação da Profissão de Educação Física e cria os respectivos Conselho Federal e Conselhos Regionais de Educação Física. Diário Oficial da União. Brasília, DF. http://www.planalto.gov.br/ccivil_03/leis/19696.htm

Lima, C. B. (2019). Dicas para elaborar seu projeto de pesquisa científica. Temas em Saúde, 2019.

Malta, D. C., Gomes, C. S., Szwarcwald, C. L., Barros, M. B. A., Silva, A. G., Prates, E. J. S., Machado, I. E., Souza Júnior, P. R. B., Romero, D. E., Lima, M. G., Damacena, G. N., Azevedo, L. O., Pina, M. F., Werneck, A. O. \& Silva, D. R. P. (2019). Distanciamento social, sentimento de tristeza e estilos de vida da população brasileira durante a pandemia de Covid-19. Saúde debate. 44, (4), 177-190.

Nahas, M. V. (2017). Atividade física, saúde e qualidade de vida: conceitos e sugestões para um estilo de vida ativo. (7a ed.). Ed. do Autor.

OMS. Organização Mundial da Saúde. Diretrizes da OMS para atividade física e comportamento sedentário: num piscar de olhos: OMS [2020]. https://apps.who.int/iris/bitstream/handle/10665/337001/9789240014886-por.pdf

Patias, N. P. \& Hohendorff, J. V. (2019). Critérios de qualidade para artigos de pesquisa qualitativa. Psicol. estud., 24, 1-14. 10.4025/psicolestud.v24i0.43536.

Pesquisa nacional de saúde: 2019: percepção do estado de saúde, estilos de vida, doenças crônicas e saúde bucal: Brasil e grandes regiões. (2020). Coordenação de Trabalho e Rendimento. IBGE, 2020.

Raimundo, A., Malta, J. \& Bravo, J. (2019). O Problema do Sedentarismo. Benefícios da Prática de Atividade Física e Exercício. Évora, Universidade de Évora. https://dspace.uevora.pt/rdpc/handle/10174/25891

Salgado, S. da S. (2017). Gestão e educação física escolar: uma mudança de postura para uma mudança de cultura. Colégio Pedro II - Revista do Departamento de Educação Física, Rio de Janeiro, v. 2(1), 49-69.

Santana, C. P., Nunes, H. A. S., Silva, A. N. \& Azeredo, C. M. (2021). Associação entre supervisão parental e comportamento sedentário e de inatividade física em adolescentes brasileiros. Ciência \& Saúde Coletiva, 26(2), 569-580, 2021.

Senhoras, E. M. (outubro de 2020). Impactos da Pandemia do COVID-19 na educação. Artigo apresentado no VII Congresso Nacional de Educação, Centro Cultural de Exposições Ruth Cardoso, Maceió-AL.

Silva, A. G., Prates, E. J. S. \& Malta, D. C. (2021). Avaliação de programas comunitários de atividade física no Brasil: uma revisão de escopo. Cad. Saúde Pública, 37(5), 1-17. 10.1590/0102-311X00277820.

Silva, A.T., Santos, L.P., Rodriguez-Añez, C.R. \& Fermino, R.C. (2021). Modelo lógico do "Programa Cidade Ativa, Cidade Saudável" de São José dos Pinhais, Paraná. Rev. Bras. Ativ. Fís. Saúde. 26, 1-6. 10.12820/rbafs.25e0193

Silva, K.S., Bandeira, A.S., Ravagnani, F.C.P., Camargo, E.M., Tenório, M.C., Oliveira, V.J.M., Santos, P.C., Ramires, V.V., Sandreschi, P.F., Hallal, P.C. \& Barbosa Filho, V.C. (2021). Educação física escolar: Guia de Atividade Física para a População Brasileira. Rev. Bras. Ativ. Fís. Saúde. 26, 1-18. $10.12820 /$ rbafs.26e0219.

Sousa, A. S., Oliveira, G. S. \& Alves, L. H. (2021). A pesquisa bibliográfica: princípios e fundamentos. Cadernos da FUNCAMP, $20(43)$ 64-83.

Souza, G. L. A. \& Silva, M. C. (2018). A relação entre o sedentarismo e a má alimentação com o aumento no índice de sobrepeso e obesidade em crianças. [Monografia Graduação, Faculdade de Ciências da Educação e Saúde, Centro Universitário de Brasília]. 
Research, Society and Development, v. 11, n. 1, e25011124731, 2022

(CC BY 4.0) | ISSN 2525-3409 | DOI: http://dx.doi.org/10.33448/rsd-v11i1.24731

Vasconcellos, M. B., Polycarpo, I. E. A. M., Santana, D. D. \& Veiga. G. V. (2021). Mudanças na obesidade, comportamento sedentário e inatividade física, entre 2010 e 2017, em adolescentes. J. Phys. Educ. 32, 1-12.10.4025/jphyseduc.v32i1.3280.

Voser, R. C., Lima, D. V., Voser, P. E. G. \& Junior, M. A.S. D. (2017). Mensuração do nível de atividade física de escolares da rede pública de ensino da cidade de pelotas-rs. Revista Brasileira de Prescrição e Fisiologia do Exercício, São Paulo. 11(70), 820-825.

World Health Organization. (2017). Healthier, fairer, safer: the global health journey. 2007-2017. Organização Mundial da Saúde. (2017). Mais saudável, mais justo, mais seguro: a jornada global da saúde, 2007-2017. 